\title{
Vibration Analysis and Optimal Design of Pneumatic Circuits Using Artificial Neural Networks
}

\author{
Şahin YILDIRIM \\ Mechatronic Engineering Department \\ Erciyes University, Faculty of Engineering \\ Kayseri, Turkey \\ sahiny@erciyes.edu.tr
}

\author{
Aslı DURMUŞOĞLU \\ Mechanical Engineering Department \\ Erciyes University, Faculty of Engineering \\ Kayseri, Turkey \\ asli@erciyes.edu.tr
}

\begin{abstract}
Pneumatic systems are commonly used in industry processes and applications of automotion. These systems are make attractive power transmission with the compressed air, because of they are economic, clear, safe and simple structured.So in this systems, as noise and vibration effects, it' $s$ undesirable situations both human health and system performances yield and working life. In this study, vibration and noise datas are obtained from two type of pneumatic systems prototype which are classify metal and non-metal materials and performed an analysis with help of this datas and used neural network which has adaptive and quick contruction.
\end{abstract}

Keywords-pneumatic circuits; vibration analysis; neural networks

\section{INTRODUCTION}

Capable of controlling the pressure of the situation that can be changed, with systems that are running air and gases, pneumatic systems are called. Pneumatic systems; clean, secure and with compressed air power transmission due to its simple structure makes it attractive. Thanks to this system, automation production is smooth, fast and controllable systems has gained importance in advancing the technology for use as. And considering the area they are used in automation technology, this importance is even more evident.

Pneumatic cylinders, reproducibility, low cost and has a wide use in industrial applications in terms of superior performance. In many cases, to achieve good control performance, nonlinear control techniques have been used. Simaui and colleagues [1] one of these techniques by using the cascade method the back electro-pneumatic position control system have worked on.

Pneumatic systems, industrial processes, and are used extensively in automation applications. However, this system must be made of the analysis of instantaneous dynamic changes.

Akkaya and his colleques [2] have been carried out the simulation of the dynamic properties of a linear pneumatic actuator system, and thus studied the effect of parameter variations of the system despite. Simulation study in the
Matlab-Simulink model was carried out using a computer program created in.

Pneumatic vibration isolation system (PVIS), effective for vibration isolation due to the performance, are widely used. Moon and Lee [3] were intended to do pvis systems modelling and sensitivity analysis. Pu and colleagues [4] in their study of the police system to adjust the damping characteristics of double-chamber pneumatic spring is used. The purpose of this adjustable pvis to improve the performance characteristics of the system.

In this study, two different separator pneumatic test and separation station for ANNS (artificial neural networks) using vibration analysis and modeling are reviewed. Minimum and maximum values for materials of different weight and pressures taken at the pressure difference cylinder pneumatic vibration the effects of vibration on the system were investigated.

\section{SEPARATOR PNEUMATIC TEST AND SEPARATION STATIONS}

\section{A. The Physical Structure Of The System}

Separator and separation PLC controlled pneumatic test stations, color, height, and reservoir samples are automatically segregated into different metal with different characteristics as to whether or not. The separation station also is controlled with two pneumatic cylinders and a PLC controller with DC motors and the movement and the separation process are provided. The samples for the determination of the properties of various sensors distance sensors inductive sensors are used.

The first test station and components of the system and separation Figure 1 seen. Sampling at this station, which is moved by a DC motor of that piece of progress is provided by the marching band. As can be seen from the figure, the first part of the tape consists of various sensors for making measurements and test. The sensors thenaccording to the results of the test process, to perform the separation process, pneumatic cylinders, and material of the channels is located. This test samples at the station and separation of white and black metal depending on whether they are pneumatic separators is parsed through to different sites 


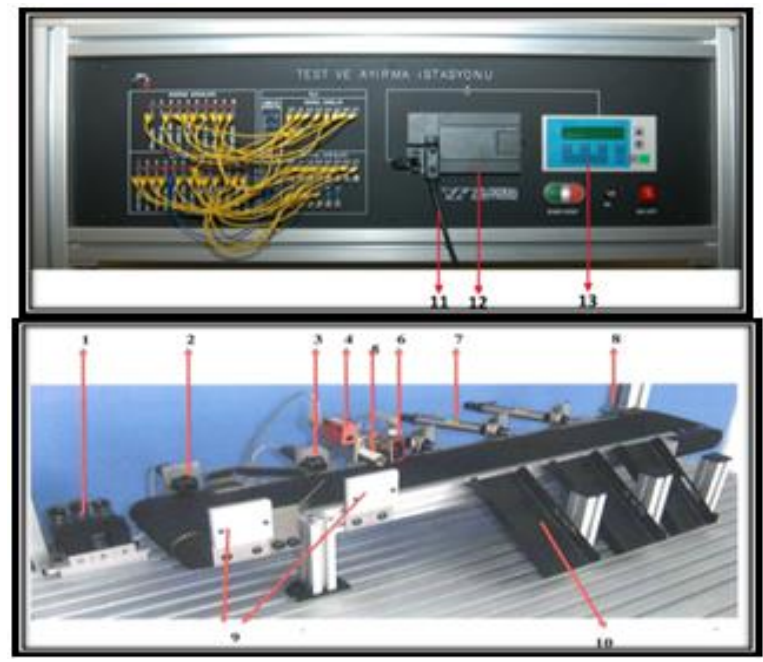

Fig 1.Test and separation Station and the system components(Mechanism 1)

Some instruments of the Figure 1, can be describes in the following;

1.Electro-pneumatic solenoid valve group

2. and 3. Optic sensors

4. The distance (heightness) sensor

5. Induktifsensor

6. Contrastsensor

7. Single acting pneumatic cylinders

8. Belt

9. Reflectors

10. Material

11. Programming cables

12. PLC

13. Operator panel

The second test seen in Figure 2, and the separation Station and the system components. At this station the progress of a DC Motor the motion of pneumatic cylinders and sample piece provided by the system. Samples placed in the starting position of the first sensor and the measurements to be made are being moved to two positions. The last point of the measurement result from the material to the channel above or below, again are pushed by a pneumatic cylinder.

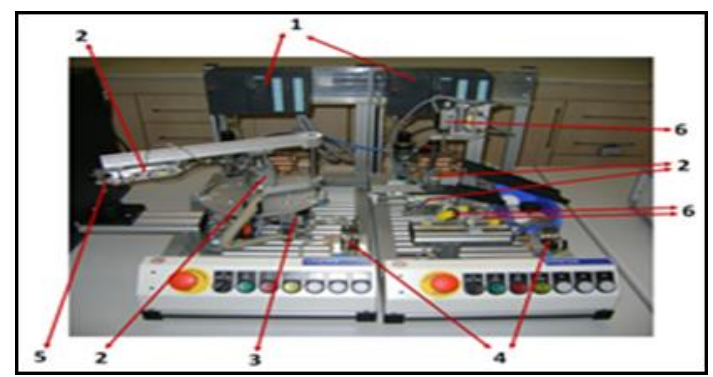

Fig 2. Test and separation Station system components(Mechanism 2)
Separator and separation PLC controlled pneumatic test stations, color, height, and as to whether or not to metal Figure 2 common system components;

1. PLC (S7-300)

2. Pneumatic Cylinders

3. DC Motor

4. Electro-Pneumatic Valve Group

5. Pneumatic Vacuum Gripper

6. Sensors

The schematic of the system structure, the structure is quite similar to the previous schematic of the station. (Fig 1.) The difference here, the system consists of two workstations. The movement of the sample for the transport of the first station, the second Station and the measurements are used for the parsing process. These two stations, and is controlled by two different PLC stations works interactively with each other.

Test specimens from this test and separation station the Black, White and whether they are properties such as metal are studied. Also there is a hole on one side of the samples. In this way the specimen is placed that will be performed to check whether or not flat or inverted.

\section{B. Mathematical Structure Of The System}

Investigated pneumatic systems, single acting cylinder, double acting cylinder and electro-pneumatic directional control valves are discussed as including three separate elements. The standard orifice using the theory of dynamic properties of elements and each element is given mathematical model of a pneumatic.

When you created the model, the following assumptions were made

- $\quad$ Air is an ideal gas.

- $\quad$ Feeding pressure (Ps) is constant

- Exhaust pressure (PR) is equal to atmospheric pressure $(\mathrm{Pa})$.

- The process is isentropic.

- Feed cylinder is constant and equal to the temperature the temperatures in the compartments in the tank.

- The dynamics of the piston valve and the hose has been neglected.

\section{Cylinder Model}

Push and pull forces single acting pneumatic cylinders can be calculated as follows. In the direction of the thrust force than the force in the direction of pull. The reason for this is due to tensile compressed air in the direction of the applied field will be smaller than the shafts. 


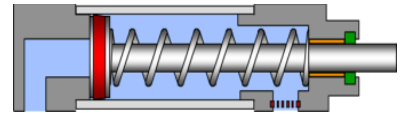

Fig 3. Single acting pneumatic cylinder [8]

Theoretical force calculation;

$$
\begin{aligned}
& A_{1}=\pi \cdot \frac{D^{2}}{4} F_{\text {thrust }}=P \cdot A_{1} \\
& A_{2}=\pi \cdot \frac{D^{2}-d^{2}}{4} F_{\text {pull }}=P \cdot A_{2}
\end{aligned}
$$

Figure 4, 4/2 directional control valve double acting cylinder controlled by a also has been given. Here $x, \dot{x}, \ddot{x}$, respectively, position, speed, acceleration $\left[\mathrm{m}, \mathrm{m} / \mathrm{s}, \mathrm{m} / \mathrm{s}^{2}\right] ; P_{1,2}$ to cylinder 1 . and 2. the pressure in the compartments $[\mathrm{Pa}] ; P_{S, R}$ supply and exhaust pressure $[\mathrm{Pa}] ; A_{1,2}$ cylinder 1 . and 2 . the area on the side of the piston $\left[\mathrm{m}^{2}\right] ; \dot{m}_{1,2}$ cylinder 1 . and 2 . on the side of the mass air flow $[\mathrm{kg} / \mathrm{s}] ; i_{\text {ref }}$ control system and valve piston weight $\mathrm{M}[\mathrm{kg}]$ represents.

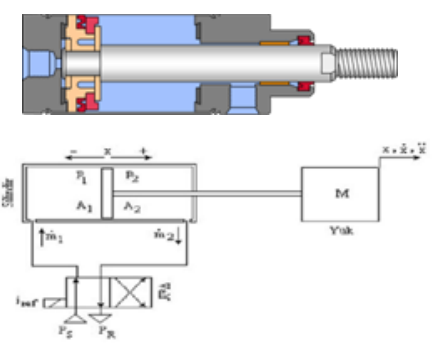

Fig 4. Double acting pneumatic cylinder [8]

The feed pressure of the valve position of which determines how the cylinder is opened to atmospheric pressure or to. The load forward $(+)$ to move the cylinder 1 . compartment opens into the feed tank. Simultaneously, the cylinder 2. the compartment is opened to the atmosphere. This condition gives rise to the pressure difference between the compartments of the cylinder. The difference in pressure increases enough to defeat friction and external forces when the piston and thus the load starts to move. The equation of motion for the cylinder is as follows [5];

$$
\begin{gathered}
F_{n e t}=P_{1} \cdot A_{1}-P_{2} \cdot A_{2} \\
M . \ddot{x}=F_{n e t}-F_{s} \\
M . \ddot{x}+F_{s}=P_{1} \cdot A_{1}-P_{2} \cdot A_{2} \\
F_{s}=f\left(\dot{x}, P_{1}, P_{2}, \ddot{x}, t\right)
\end{gathered}
$$

Equation (4), $F_{n e t}$ the net force on the system due to the difference in pressure, if the force of friction is $F_{S}$.

Volts mass air flow compressed from the cylinder element for the input variables, the position and velocity of the piston. A cylinder 1 . output variables and 2 . the pressure in the compartments. The relationships between pressure and flow are given below [6];

$$
\dot{P}_{1}=\frac{k}{\frac{L}{2}+x+x_{d}}\left[-P_{1} \cdot \dot{x}+\frac{R T}{A_{1}} \dot{m}_{1}\right]
$$

$$
\dot{P}_{2}=\frac{k}{\frac{L}{2}-x+x_{d}}\left[P_{2} \cdot \dot{x}+\frac{R T}{A_{1}} \dot{m}_{2}\right]
$$

\section{Valve Model}

Valve element from the previous element for pressurized air and an electrical signal input of the variable that is set by the location of the valve. As output variables depending on the position of the valve cylinder 1 . pane or 2 . entered into the pane, or the compressed mass air flow.

The port area $A_{v}$ valve, and valve discharge coefficient $C_{d}$ constants, such as input system can be considered as constant. The relationships between valve input and output variables are given in the following equations [5].

The piston cylinder forward (+) movement in the case;

$$
\begin{aligned}
& \dot{m}_{1}=C_{d} A_{v} \frac{P_{s}}{\sqrt{T}} \phi\left(P_{s}, P_{1}\right) \\
& \dot{m}_{2}=C_{d} A_{v} \frac{P_{2}}{\sqrt{T}} \phi\left(P_{2}, P_{a}\right)
\end{aligned}
$$

where;

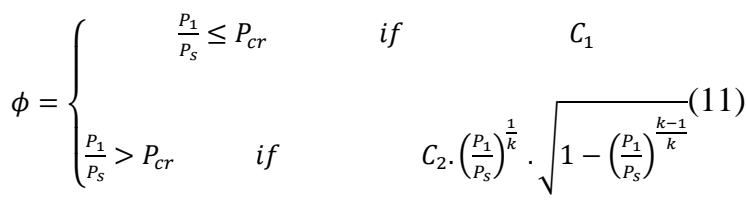

The piston cylinder backward (-) in the case of progress;

$$
\begin{aligned}
& \dot{m}_{1}=C_{d} A_{v} \frac{P_{1}}{\sqrt{T}} \phi\left(P_{1}, P_{a}\right) \\
& \dot{m}_{2}=C_{d} A_{v} \frac{P_{s}}{\sqrt{T}} \phi\left(P_{s}, P_{2}\right)
\end{aligned}
$$

where;

$$
\begin{aligned}
& \phi=\left\{\begin{array}{ccc}
\frac{P_{a}}{P_{1}} \leq P_{c r} & \text { if } & C_{1} \\
\frac{P_{2}}{P_{s}}>P_{c r} & \text { if } & C_{2} \cdot\left(\frac{P_{1}}{P_{s}}\right)^{1 / k} \cdot \sqrt{1-\left(\frac{P_{1}}{P_{s}}\right)^{(k-1) / k}}
\end{array}\right. \\
& C_{1}=\sqrt{\frac{k}{R}\left(\frac{2}{k+1}\right)^{\frac{k+1}{k-1}} ; C_{2}=\sqrt{\frac{2 k}{R(k-1)}} ; P_{c r}=\left(\frac{2}{k+1}\right)^{\frac{k}{k-1}}}
\end{aligned}
$$

where $k$ is the specific heat ratio, $R$ the gas constant critical pressure and $P_{c r}, C_{1,2}$ the mass flow parameter.

\section{EXPERIMENTAL WORK}

Two different values are measured at different pressures for different test and separation station the vibrational modeling artificial neural networks are reviewed. Stations for testing and sorting the material in 3 different weights with the vibration values are measured at minimum and maximum pressures in pneumatic cylinder and has performed analysis and modeling. While modeling algorithms that belong to 5 different artificial neural networks algorithms that give the best and worst results are presented in graphs and tried.

Test and separation station the first pneumatic separator for black, white and metal $(25 \mathrm{~g}, 15 \mathrm{~g}, 10 \mathrm{~g})$ single acting 
cylinder used for separating three materials, (1., 2. and 3. measurement points) 2 bar working pressure and 6 bar vibration values were taken.

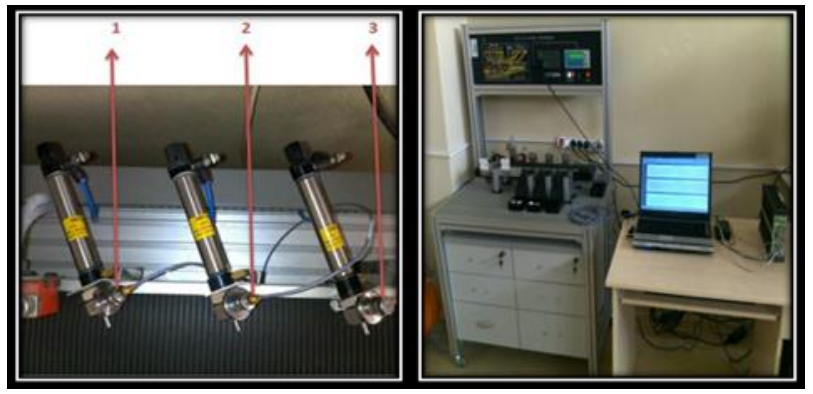

Fig 4. Separator pneumatic test and separation station and an experimental setup for measuring the acceleration (1).

Second separator pneumatic test and separation station 3 different weights $(50 \mathrm{~g}, 100 \mathrm{~g}, 240 \mathrm{~g})$ material for a working pressure of 2 bar and 6 bar, two double acting cylinders (1. and 2. measuring point) and DC motor (3. measuring point through the vibration values were taken.

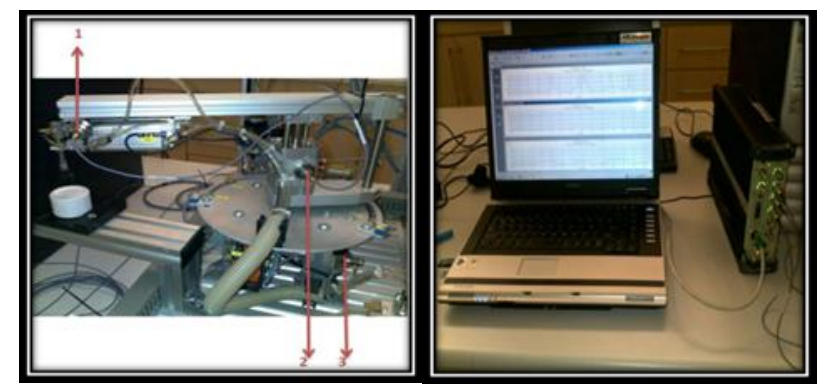

Fig 5. Separator pneumatic test station and an experimental setup for measuring the acceleration and separation (2).

\section{ARTIFICIAL NEURAL NETWORKS (ANN)}

Separator pneumatic test stations, experimental setups and separation of the Bruel - Kjaer portable and multi-channel pulse data has been received and with the help of accelerometer sensors using artificial neural networks by using modeling and analysis conducted. In Figure 6 The Block diagram of this situation are given.

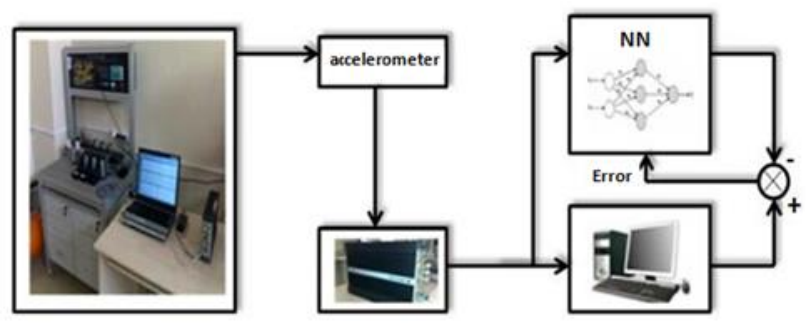

Fig 6. Block diagram of experimental system

The most typical form of the NN structure, the cell generated with the model of the layers to be assembled can be installed with the sequential format. A neural network model used is shown in Figure 7.

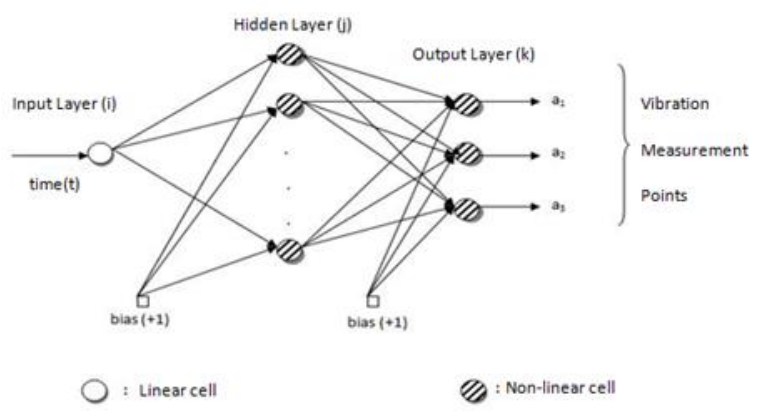

Fig 7. ANN's schematic representation

Here the applied layer to the input layer of the input signal, the output signal of the receipt of the response layer is called the output layer. In the present study, a linear cell in the input layer, hidden and output layers, respectively, ten and three nonlinear cell was used. Given the network obtained for the training, $70 \%$, for the test phase, $30 \%$ were used. ANN's data to be used for the purpose of testing for the stable structure of the network during the training phase were not used.

\section{A. Back Propagation Algorithm}

The most common applications used in the learning algorithm. Most preferred because it can be easily verified mathematically easy to understand and the learning algorithm. Reverse this algorithm to reduce errors back propagation got its name because of working from the output to input [7].

Back propagation algorithm, the slope is decreasing and MLP' $s$ the most basic learning algorithm used in training. In this algorithm the weights between the elements $i$ and $j$ with processing times $\Delta w_{j i}(t)$, the change in is calculated. This expression,

$$
\Delta w_{j i}(t)=\eta \delta_{j} x_{i}+\alpha \Delta w_{j i}(t-1)
$$

as it is provided. The equality (16) $\eta$ the learning coefficient, momentum coefficient $\alpha$ and of belonging to any neuron $j$ in the output layer Dec or $\delta_{j}$ is a factor. For the output layer, this factor is provided in the following manner;

$$
\delta_{j}=\frac{\partial f}{\partial n e t_{j}}\left(y_{j}^{t}-y_{j}\right)
$$

where,

$$
n e t_{j}=\sum x_{j} w_{j i}
$$

and $y_{j}^{t}, j$ the target output of the element processor. Dec factor for the neurons in the layers;

$$
\delta_{j}=\left(\frac{\partial f}{\partial n e t_{j}}\right) \sum w_{q i} \delta_{q}
$$

\section{B. QuickPropagation Algorithm (QP)}

Quick propagation algorithm, developed by Fahlman and newton method based on a heuristic learning algorithm used for MLP training. Propagation tests are usually fast when compared with other techniques the performance of the algorithm is pretty good. This algorithm gives better results in less data problems with the noise level especially [7]. 
This algorithm is neglected and the weight in acceleration and clipped the $k-1$ from $k$ up to in weight change,

$$
\Delta w(t)=\varepsilon L(t)+\alpha Q(t)
$$

with the formula is obtained. Here $\varepsilon$ is the learning coefficient, and momentum coefficient $\alpha$ is. Here to update the weight function, the delta function and participates in the processing of the acceleration due to gravity, the coefficient of weight.

$$
w(t)=(1-\delta) w(t-1)+\Delta w(t)
$$

Here $\delta$ is the coefficient acceleration. Finally, if the weight is too small, 0 is clipped by taking. $|w(t)|<K$ if $w(t)=0$ is taken. Here, the weight factor $K$ crop [6].

\section{Delta-Bar-Delta Algorithm}

Delta-Bar-Delta multi-layered perseptron convergence of the connection weights in a heuristic approach that is used to increase the speed. In experimental studies, the weight of each dimension in terms of space have shown that all the error surface can be completely different. To explain the changes in the error surface, especially the coefficient for each link of the network should have own learning. The appropriate step size for the size weight only the weight of this thought may not be suitable for all sizes [7]. The standard back propagation algorithm, the slope component is provided in the following way,

$$
\partial(k)=\frac{\partial E(k)}{\partial w(k)}
$$

Here $E(k)$ at the time of the error value $k, w(k)$ and the weight of the connection $\delta(k)$ illustrates the slope of the change in weight component. The standard back propagation algorithm of the Connection Weight;

$$
w(k+1)=w(k)+\alpha \delta(k)
$$

will be updated as. Here $\alpha$ is a learning constant coefficient. DBD learning rule, the learning rate for each connection, the variable $\alpha(k)$ is assigned to, and update of the Connection Weight;

$$
w(k+1)=w(k)+\alpha(k) \delta(k)
$$

\section{SimUlation RESUlts}

The scope of work testing and sorting stations in five experimental setups for vibration analysis neural network algorithm was used. Experimentally obtained vibration data is taken as the desired value. At Station 107 the first test for vibration and separation of the network the training data, the vibration data were used in the testing phase 50. 175 the second test and separation station of the network during training, vibration data, vibration data were used for the test phase 90. ANN' s evaluation of the accuracy of modeling with the data to be used during training for the data to be used in the test phase is different.

Vibration analysis for the study of back propagation algorithm, batch algorithm, backprop, delta-bar-delta algorithm, quickpropagation (fast propagation) algorithm and prop (flexible propagation) algorithm was used. The first test and separation station the best results for fast propagation algorithm, while the worst results delta-bar-delta algorithm were obtained.

The second test and separation station for best results the worst results have been obtained in the back propagation

\begin{tabular}{|c|c|c|c|c|c|c|c|}
\hline \multirow[t]{2}{*}{ Algoritm Type } & \multirow{2}{*}{$\begin{array}{l}\text { Leaming Rate } \\
(\eta)\end{array}$} & \multirow{2}{*}{$\begin{array}{l}\text { Momectum } \\
\text { Coefficient } \\
\text { (a) }\end{array}$} & \multirow{2}{*}{$\begin{array}{l}\text { leration } \\
\text { Nimber }\end{array}$} & \multicolumn{3}{|c|}{ Cell Nimber } & \multirow[b]{2}{*}{ Error } \\
\hline & & & & Input & Hidden & Output & \\
\hline $\begin{array}{c}\text { Back- } \\
\text { Propagation }\end{array}$ & 0.01 & 0.4 & 1.000 .000 & 1 & 10 & 3 & 0.163 \\
\hline Detta-Bar-Detita & 0.01 & 0.4 & 1.000 .000 & 1 & 10 & 3 & 0.578 \\
\hline QuickProp & $0.0 !$ & 0.4 & 1.000 .000 & 1 & 10 & 3 & 0.148 \\
\hline
\end{tabular}
algorithm and is still the delta-bar-delta algorithm were obtained.

TABLE I. CHARACTERSTICS OF DIFFERENT LEARNING ALGORITHMS İN FEEDFORWARD ANNS

A. Max. and min. pressure in the simulation results (Mechanism 1)

Max. and min. the rapid pressure propagation algorithm and Delta - bar - Delta the results of the simulation figure 8,9, 10 and 11 are given.

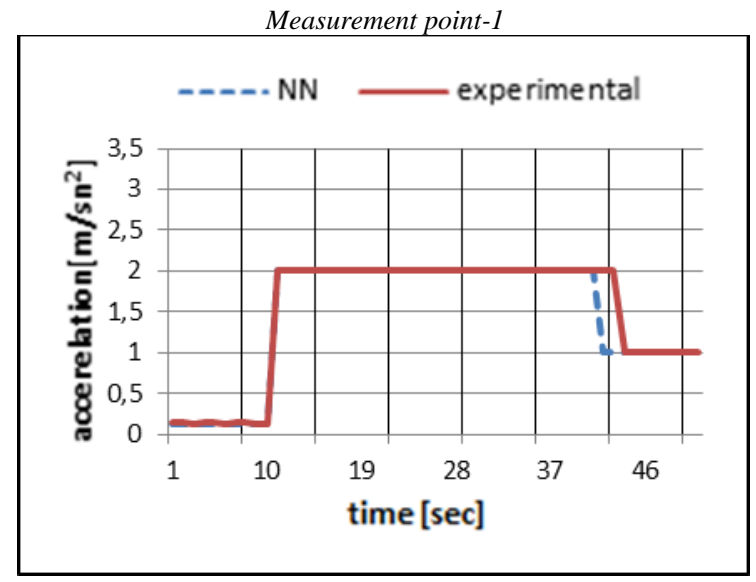

Measurement point-2

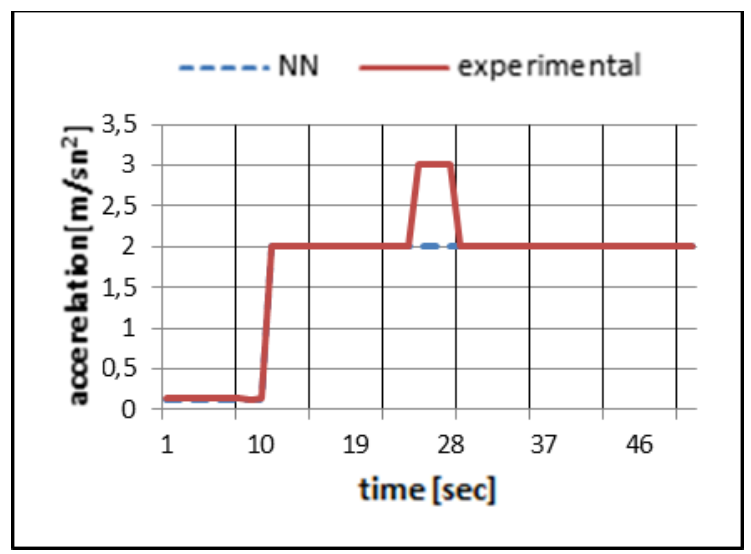


Measurement point-3

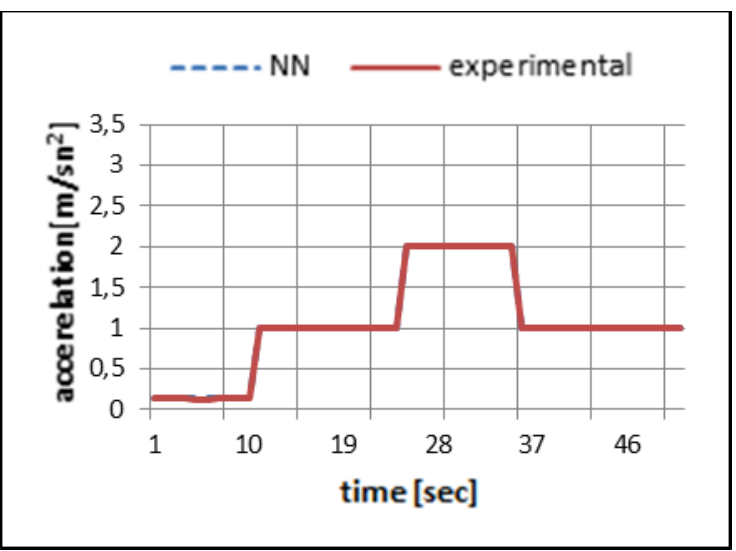

Fig 8. Different measurement points max.experimental and neural network results of pressure(fast-propagation algorithm)

In the above chart max. rapid pressure propagation algorithm results are given. Figure. 8. as seen, the highest vibration 2. ANN and occurred largely at the point of measurement to the desired answer uncovered. 1. and 3. the vibration values at the measurement points smaller neural network has been able to track the desired values.
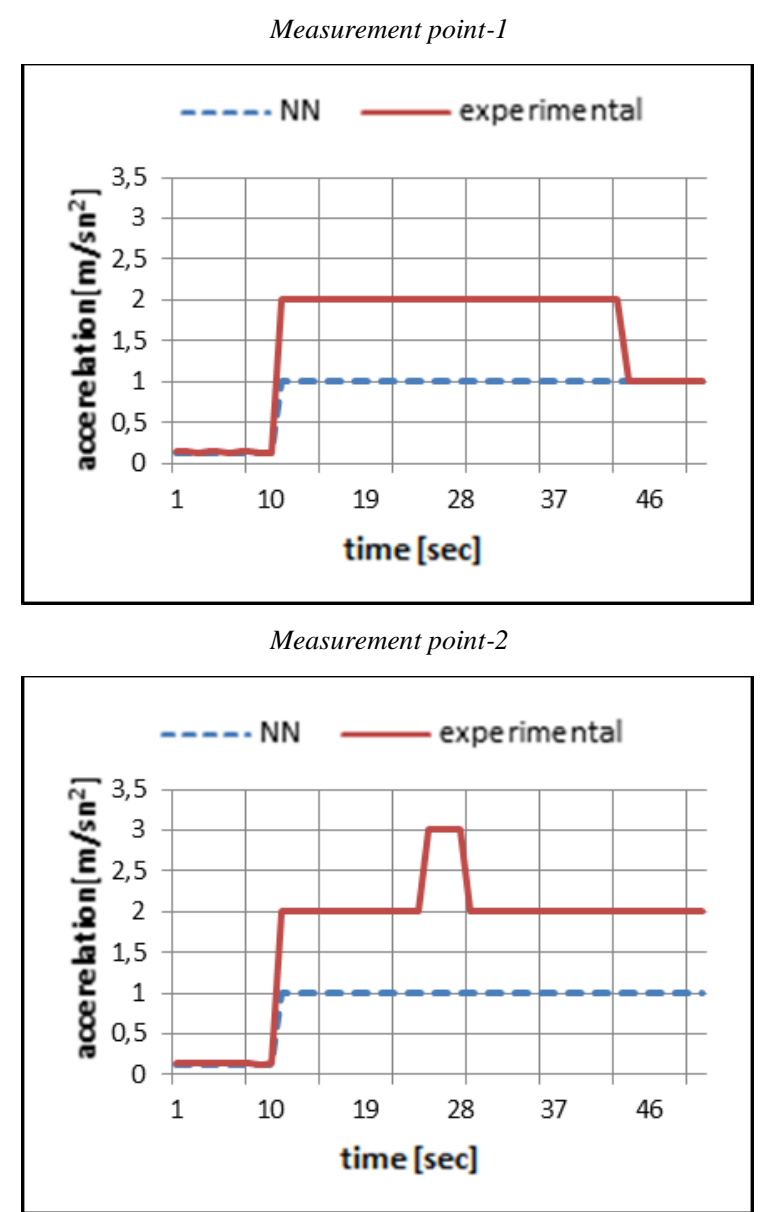

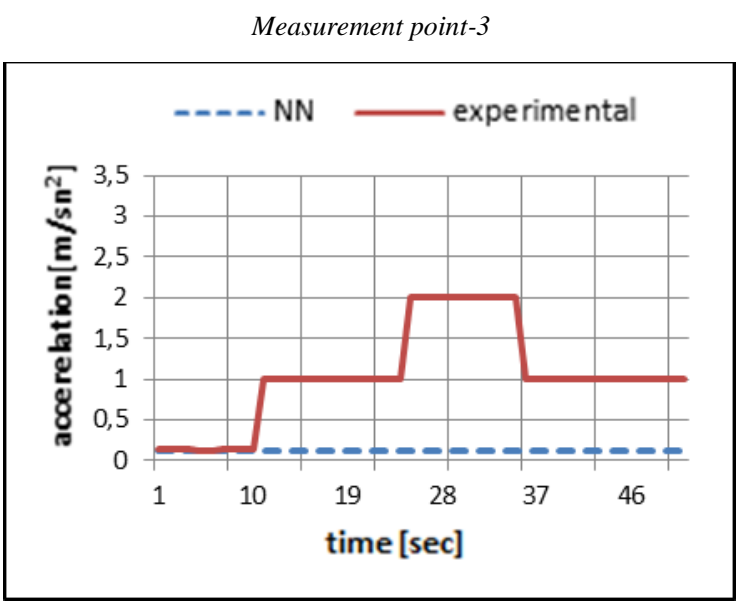

Fig 9. Different measurement points max.pressure-experimental and neural network results(Delta-bar-delta algorithm)

Delta-bar-delta neural network algorithm with three measurement points in the desired response could not catch up. This algorithm ANN' $s$ in the test phase the correct percentage is $15 \%$.

Measurement point-1

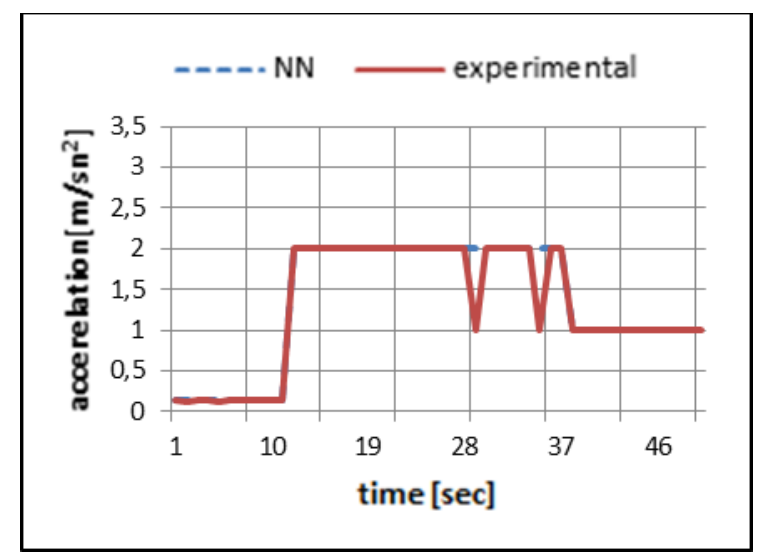

Measurement point-2

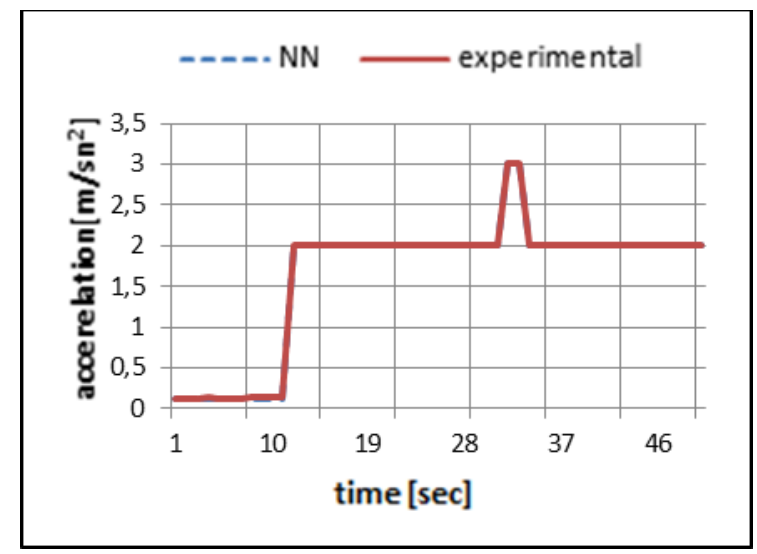


Measurement point-3

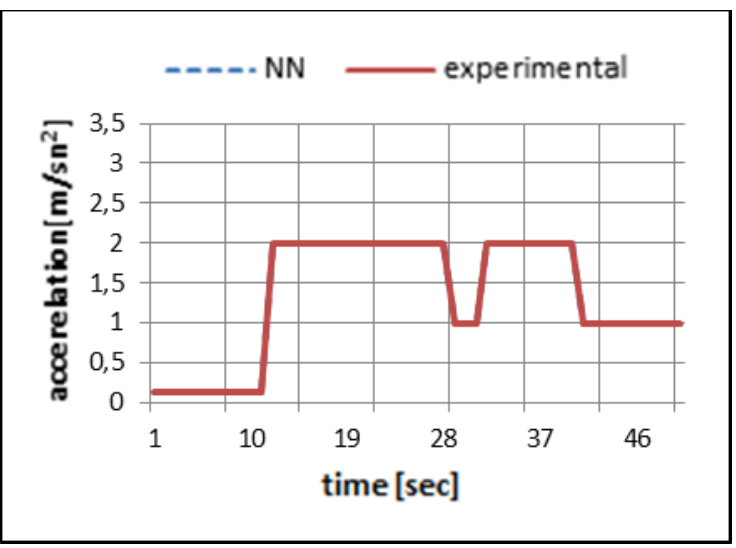

Fig 10. Different measurement points for min.experimental and neural network results of pressure(fast-propagation algorithm)

Figure 10, the maximum vibration value, as shown in 2 . were taken from the measurement point. Fast-propagation algorithm neural network gave the desired answer. 1. despite the lower value of the vibration measurement at the point of sudden NN could not catch up with the changes at the points where the desired values. 3. the measurement point of the NN, the desired answer has followed.

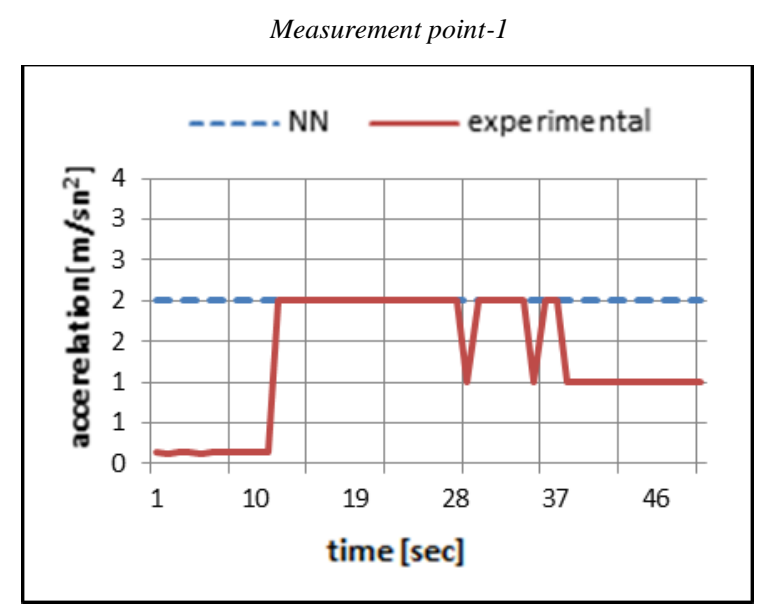

Measurement point-2

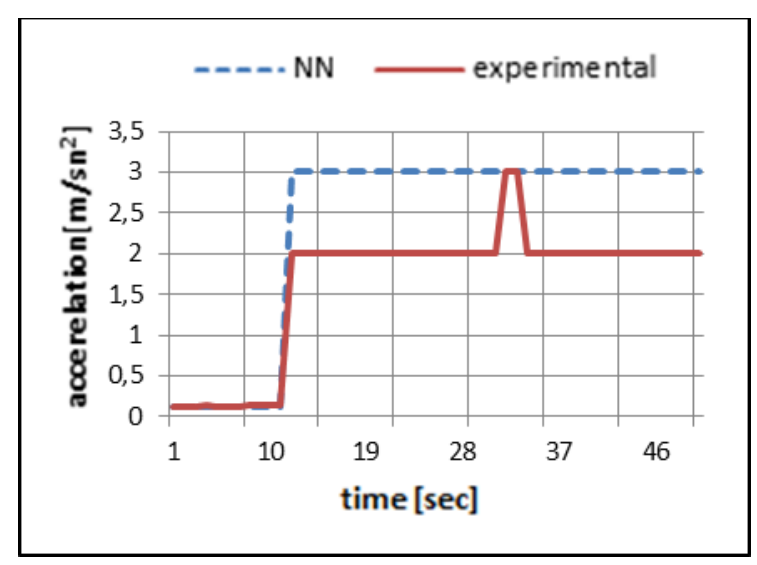

Measurement point-3

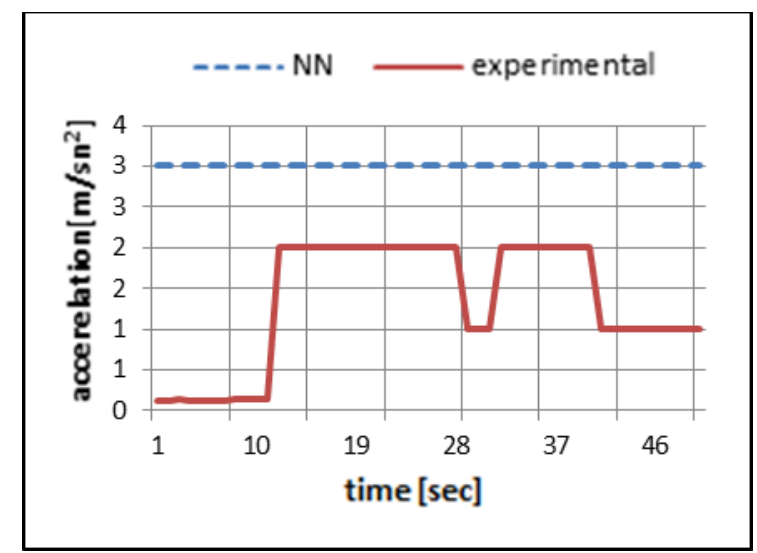

Fig 11. Different measurement points for min.pressure-experimental and neural network results(Delta-bar-delta algorithm)

Delta-bar-delta algorithm with neural network has been able to track the desired values. 1. and 3. despite the low frequency value at the point of measurement, neural network did not get the desired response. ANN with this algorithm' $s$ accuracy percentage of $2 \%$ due to remained.

B. Max. and min. pressure in the simulation results (Mechanism 2)

Max. and min. pressure propagation algorithm back propagation and Delta - bar - Delta the results of the simulation figure $12,13,14$ and 15 are given.

Measurement point-1

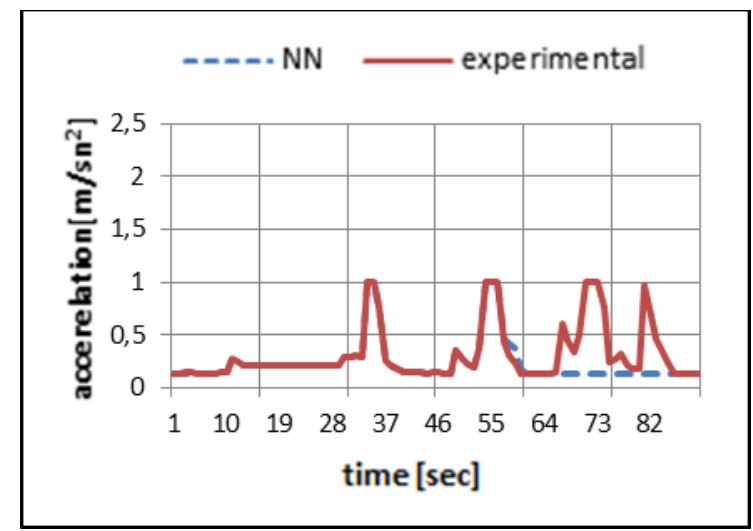

Measurement point-2

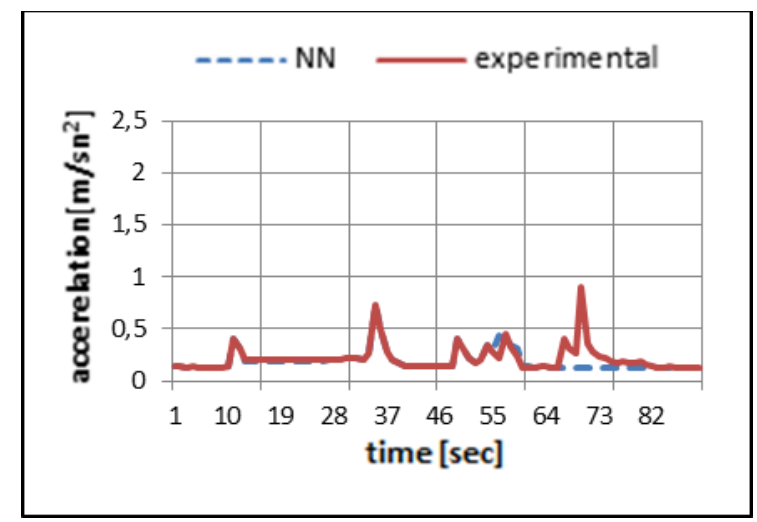




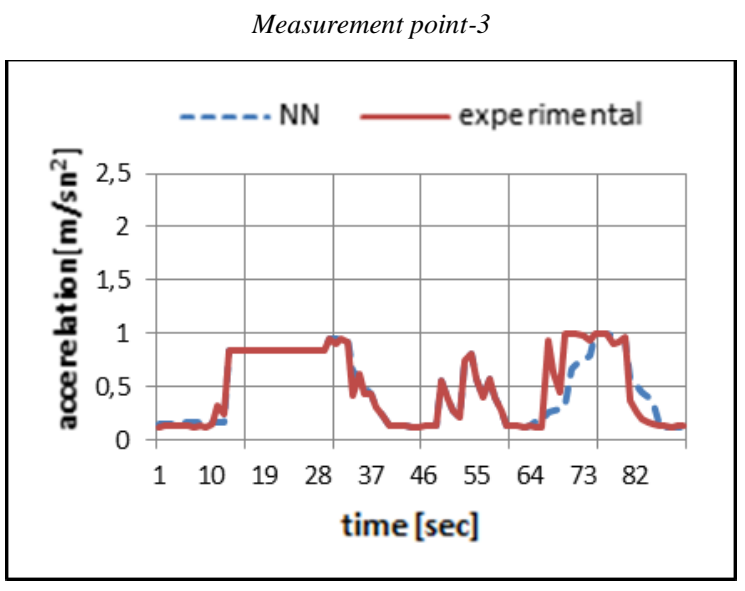

Fig 12. Different measurement points max .experimental results of pressure and artificial neural network(back propagation algorithm)

In the above chart max. back propagation algorithm results in pressure are given. 1. with the increase in pressure between measurement points change in vibration datahas been observed. ANN, this has largely followed the desired data at the point of measurement. 2. and 3. very large differences were observed in the measurement points. ANN, this algorithm is $80 \%$ and achieved an accuracy rate of.

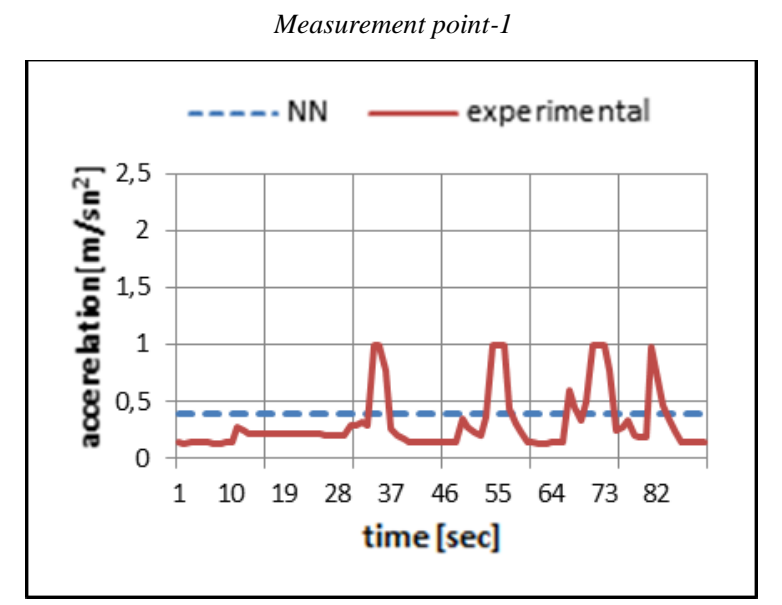

Measurement point-2

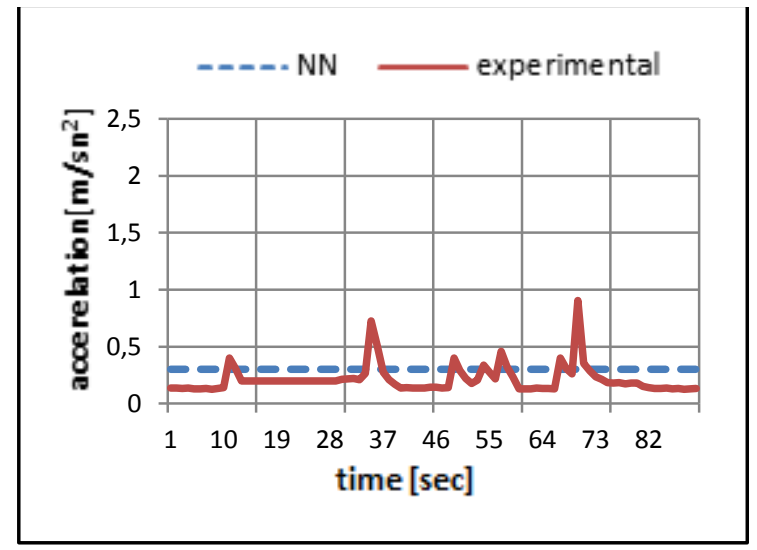

Measurement point-3

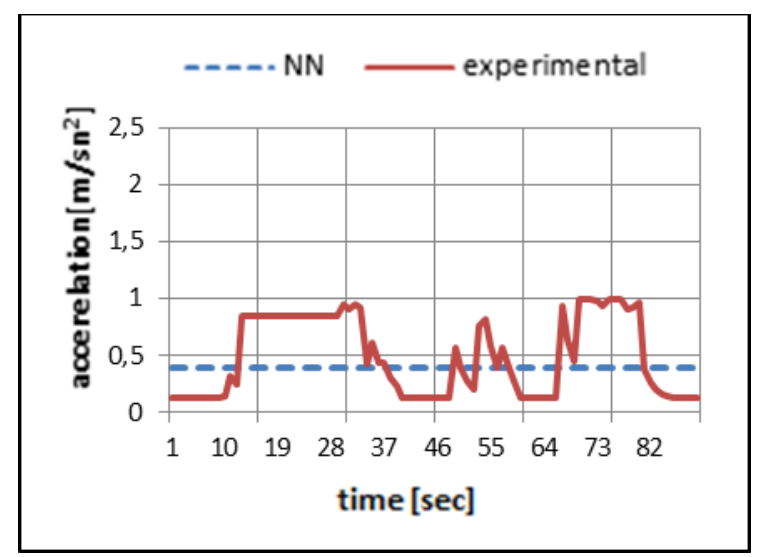

Fig 13. Different measurement points max.pressure-experimental and neural network results(Delta-bar-delta algorithm)

Delta-bar-delta algorithm, neural network the output data to the desired values at three measurement points has been able to follow the effect of vibration.

Measurement point-1

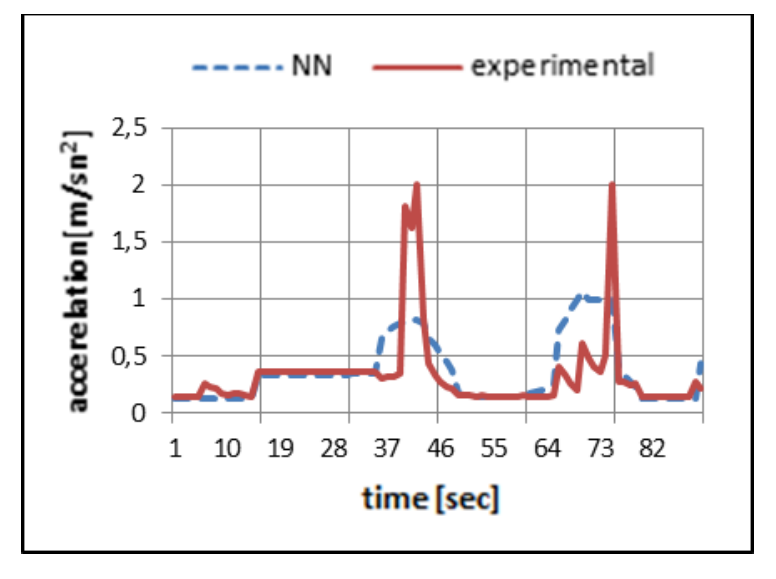

Measurement point-2

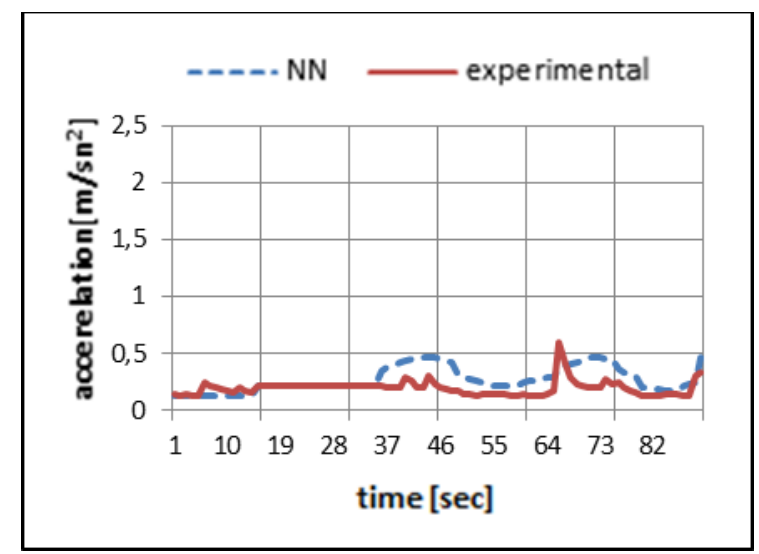




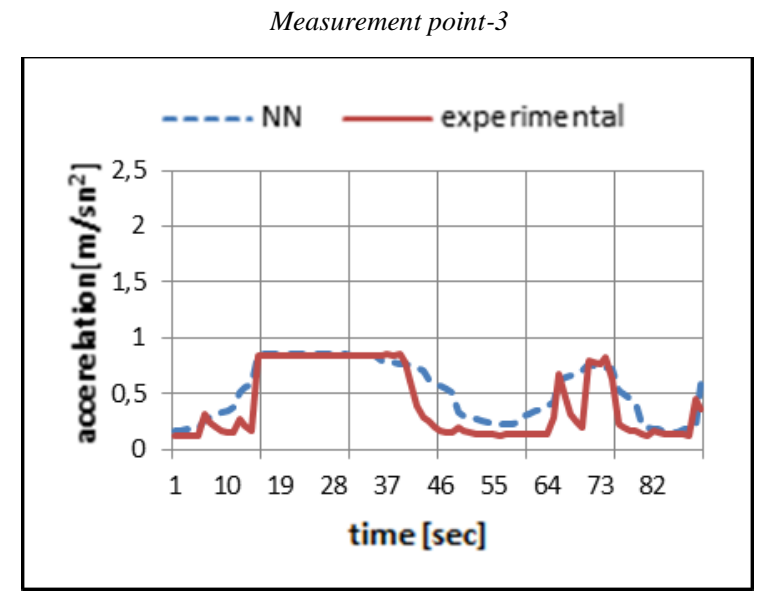

Fig 14. Different measurement points min..pressure-experimental and neural network results (Backpropagation algorithm )

In the above graph, pressure, results of back propagation algorithm are given. ANN, at the points where the vibration is able to track sudden changes of the desired values. Apart from that ANN' s accuracy the percentage of the algorithm for $50 \%$. Where 2 values are lower vibration.relatively good results were obtained at the point of measurement.

Measurement point-1

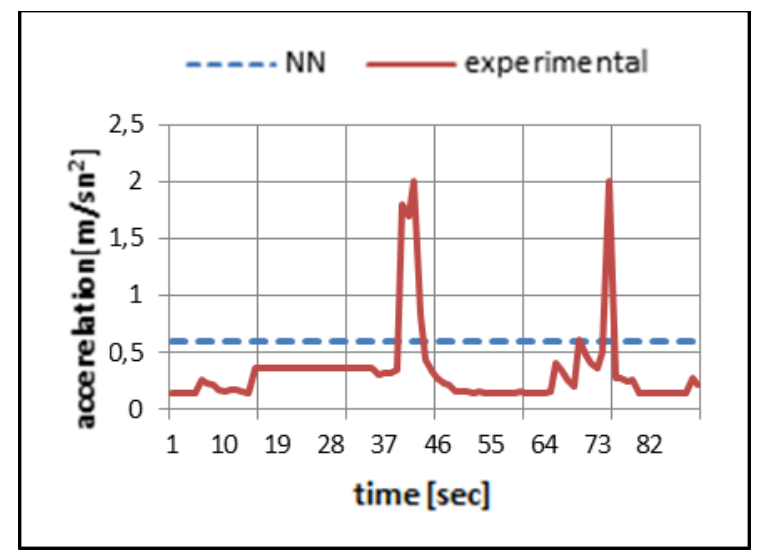

Measurement point-2

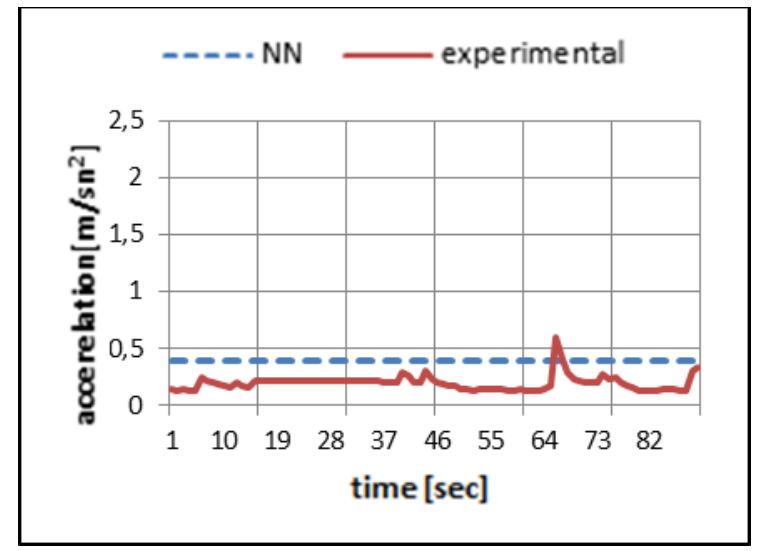

Measurement point-3

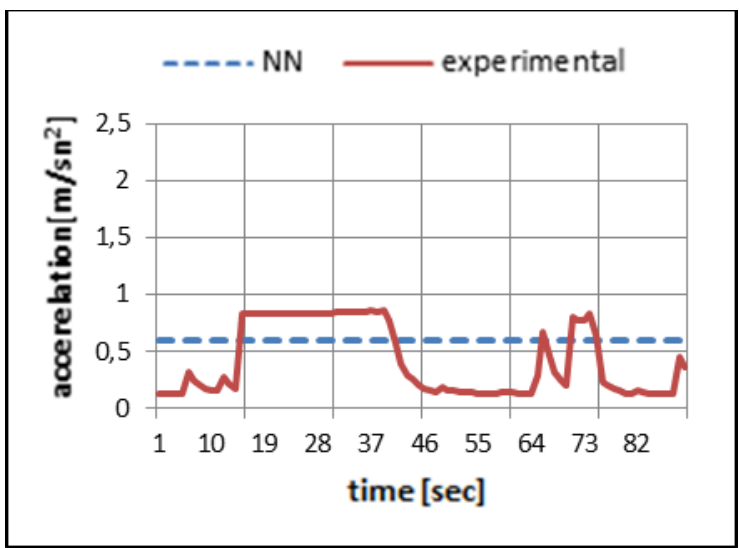

Fig 15. Different measurement points for min.pressure-experimental and neural network results(Delta-bar-delta algorithm)

As seen in Figure 15, the largest value of 1 vibration were obtained at the measurement point. ANN, delta-bar-delta algorithm did not give the desired result. ANN' s displayed a stable image output data. ANN's the accuracy rate is almost negligible.

\section{CONCLUSIONS}

In this study, two different test stations, PLC controlled pneumatic separator and the separation of test sets from different points of acceleration and vibration analysis using neural network modeling with noise effects was carried out. As a result, the vibration occurring at different working pressures and pressure changes in pneumatic systems and reviewed the effect of vibration was investigated.

Experimental and five different algorithm by using simulation results obtained are evaluated, the fast propagation algorithm and in the back propagation algorithm simulation results obtained for the same behavior experimental results tribute capability, the algorithms the vibration parameters can be used in real-time.

\section{REFERENCES}

[1] Smaoui, M., Brun, X., Thomasset, D., 2006. A study on tracking position control of an electropneumatics system using backstepping design. Control Engineering Practice, 14: 923-933.

[2] Akkaya, A. V., Sevilgen, S. H., Erdem, H. H., Çetin, B., 2005. Simulink kullanarak bir pnömatik sistemin simülasyonu. Doğuş Üniversitesi Dergisi, 6 (2): 155-162.

[3] Moon, J., Lee, B., 2010. Modelling and sensitivity anaysis of a pneumatic vibration isolation system with two air chambers. Mechanism and Machine Theory, 45: 1828-1850.

[4] Pu, H., Luo, X., Chen, X., 2011. Modelling and analysis of dualchamber pneumatic spring with adjustible damping for precision vibration isolation. Journal of Sound and Vibration, 330: 3578-3590.

[5] Karpenko, M., Sepehri, N., 2002. Neural network classifiers applied to condition monitoring of a pneumatic process valve actuator. Engineering Applications of artificial Intelligence, 15: 273-283.

[6] Sorli, M., Gastaldi, L., Codina, E., Heras, S., 1999. Dynamic analysis of pneumatic actuators. Simulation Practice and Theory, 7: 589-602.

[7] Sağıroğlu, Ş., Beşdok, E., Erler, M., 2003. Mühendislikte Yapay Zeka Uygulamaları-1. Ufuk Yayıncılık, Kayseri, $426 \mathrm{~s}$.

[8] MEGEP, 2007. Temel Pnömatik. Milli Eğitim Bakanlığı, Ankara, 75 s. 